\title{
Outflows of hot molecular gas in ultra-luminous infrared galaxies mapped with VLT-SINFONI
}

\author{
B. H. C. Emonts ${ }^{1,2}$, L. Colina ${ }^{1}$, J. Piqueras-López ${ }^{1}$, S. Garcia-Burillo ${ }^{3}$, M. Pereira-Santaella ${ }^{4}$, S. Arribas ${ }^{1}$, \\ A. Labiano ${ }^{1}$, and A. Alonso-Herrero ${ }^{1}$ \\ ${ }^{1}$ Centro de Astrobiología (INTA-CSIC), Ctra de Torrejón a Ajalvir, km 4, 28850 Torrejón de Ardoz, Madrid, Spain \\ e-mail: bjornemonts@gmail.com \\ 2 National Radio Astronomy Observatory, 520 Edgemont Road, Charlottesville, VA 22903, USA \\ 3 Observatorio Astronómico Nacional (OAN), Observatorio de Madrid, Alfonso XII, 3, 28014 Madrid, Spain \\ ${ }^{4}$ Department of Physics, University of Oxford, Keble Road, Oxford OX1 3RH, UK
}

Received 5 July 2017 / Accepted 16 August 2017

\begin{abstract}
We present the detection and morphological characterization of hot molecular gas outflows in nearby ultra-luminous infrared galaxies (ULIRGs), using the Spectrograph for Integral Field Observations in the Near Infrared (SINFONI) on the Very Large Telescope (VLT). We detect outflows observed in the $2.12 \mu \mathrm{m} \mathrm{H}_{2} 1-0 \mathrm{~S}(1)$ line for three out of four ULIRGs that we analyzed, namely IRAS 12112+0305, IRAS 14348-1447, and IRAS 22491-1808. The outflows are mapped on scales of 0.7-1.6 kpc, show typical outflow velocities of $300-500 \mathrm{~km} \mathrm{~s}^{-1}$, and appear to originate from the nuclear region. The outflows comprise hot molecular gas masses of $M_{\mathrm{H}_{2}(\text { hot })} \sim 6-8 \times 10^{3} M_{\odot}$. Assuming a hot-to-cold molecular gas mass ratio of $6 \times 10^{-5}$, as found in nearby luminous infrared galaxies, the total (hot + cold) molecular gas mass in these outflows is expected to be $M_{\mathrm{H}_{2} \text { (tot) }} \sim 1 \times 10^{8} M_{\odot}$. This translates into molecular mass outflow rates of $\dot{M}_{\mathrm{H}_{2} \text { (tot) }} \sim 30-85 M_{\odot} \mathrm{yr}^{-1}$, which is a factor of a few lower than the star formation rate in these ULIRGs. In addition, most of the outflowing molecular gas does not reach the escape velocity of these merger systems, which implies that the bulk of the outflowing molecular gas is re-distributed within the system and thus remains available for future star formation. The fastest $\mathrm{H}_{2}$ outflow is seen in the Compton-thick AGN of IRAS 14348-1447, reaching a maximum outflow velocity of $\sim 900 \mathrm{~km} \mathrm{~s}^{-1}$. Another ULIRG, IRAS 17208-0014, shows asymmetric $\mathrm{H}_{2}$ line profiles different from the outflows seen in the other three ULIRGs. We discuss several alternative explanations for the line asymmetries in this system, including a very gentle galactic wind, internal gas dynamics, low-velocity gas outside the disk, or two superposed gas disks. We do not detect the hot molecular counterpart to the outflow previously detected in $\mathrm{CO}(2-1)$ in IRAS 17208-0014, but we note that our SINFONI data are not sensitive enough to detect this outflow if it has a small hot-to-cold molecular gas mass ratio of $\lesssim 9 \times 10^{-6}$.
\end{abstract}

Key words. galaxies: interactions - galaxies: individual: IRAS 12112+0305 - galaxies: individual: IRAS 14348-1447 galaxies: individual: IRAS 17208-0014 - galaxies: individual: IRAS 22491-1808 - ISM: jets and outflows

\section{Introduction}

Ultra-luminous infrared galaxies (ULIRGs) are dust-enshrouded galaxies with massive starbursts and often a deeply buried active galactic nucleus (AGN), which may have been triggered by a gas-rich galaxy merger (Sanders \& Mirabel 1996). ULIRGs emit the bulk of their radiation at mid- and far-IR wavelengths $\left(L_{\mathrm{IR}} \geq 10^{12} L_{\odot}\right)$, where light from the starburst and AGN is absorbed and re-radiated by dust. While ULIRGs are rare in the nearby Universe and contribute at most a few percent of the infrared emission at low redshifts (Soifer \& Neugebauer 1991), they are major contributors to the star-formation activity of the Universe at high redshifts (e.g., Le Floc'h et al. 2005; Pérez-González et al. 2005; Caputi et al. 2006; Magnelli et al. 2013; Novak et al. 2017). Thus, studying the evolution of ULIRGs at low redshifts, where we can resolve the physical processes that drive these systems, is crucial for understanding the dusty, IR-luminous phase in the evolution of galaxies throughout the Universe.

A crucial aspect in the evolution of ULIRGs has been the discovery of massive gas outflows driven by starburst or AGN activity. These outflows have been extensively studied in both ionized and neutral gas (e.g., Heckman et al. 1990; Lehnert \& Heckman 1996; Rupke \& Veilleux 2011, 2013a, 2015; Westmoquette et al. 2012; Bellocchi et al. 2013; Morganti et al. 2013; Rodríguez Zaurín et al. 2013; Feruglio et al. 2015; Arribas et al. 2014; Cazzoli et al. 2014, 2016). With the discovery of outflows of neutral gas also came the realization that the mass, momentum, and energy of galactic winds and AGN-driven outflows are much higher in the neutral than in the ionized phase (Morganti et al. 2005; Emonts et al. 2005; Rupke \& Veilleux 2013a; Mahony et al. 2013). However, the full impact of outflows in ULIRGs has only been revealed with the discovery of massive outflows of molecular gas.

Molecular hydrogen is the raw fuel for star formation, and studies have revealed that molecular outflows can halt star formation, at least in the central regions of ULIRGs. These studies focused on the coldest phase of molecular outflows, by using strong tracers like $\mathrm{CO}$ and $\mathrm{OH} 119 \mu \mathrm{m}$ (e.g., Feruglio et al. 2010; 2015; Fischer et al. 2010; Chung et al. 2011; Sturm et al. 2011; Spoon et al. 2013; Veilleux et al. 2013; Dasyra \& Combes 2012; Dasyra et al. 2014; Cicone et al. 2014; Sakamoto et al. 2014; García-Burillo et al. 2015; Aalto et al. 2015; Pereira-Santaella et al. 2016; González-Alfonso et al. 2017). Direct measurements of molecular hydrogen in outflows 
rely on detecting warm and hot $\mathrm{H}_{2}$ emission in the mid- to near-IR (e.g., Valentijn \& van der Werf 1999; Ogle et al. 2010; Dasyra \& Combes 2011; Guillard et al. 2012). Because these $\mathrm{H}_{2}$ lines are relatively weak compared to $\mathrm{CO}$, studies of $\mathrm{H}_{2}$ outflows are limited to low redshift galaxies (e.g., van der Werf et al. 1993; Tadhunter et al. 2014; Hill \& Zakamska 2014), and only few studies exist that provide information on the geometry of spatially extended $\mathrm{H}_{2}$ outflows (Rupke \& Veilleux 2013b; Emonts et al. 2014; Dasyra et al. 2015; Pereira-Santaella et al. 2016). However, the detection of $\mathrm{H}_{2}$ in massive galaxies at $z \sim 2$ with the Spitzer Space Telescope shows the potential for studying the hot molecular Universe with the James Webb Space Telescope (JWST; Ogle et al. 2012; Guillard et al. 2015).

In this paper, we study the complex kinematics of the hot molecular $\mathrm{H}_{2}$ gas in a sample of four nearby ULIRGs $(0.04<$ $z<0.08)$. By performing a multi-Gaussian decomposition of the $2.1218 \mu \mathrm{m} \mathrm{H}_{2} 1-0 \mathrm{~S}(1)$ line, we discovered that three of these systems show evidence for an outflow of hot molecular gas, which we resolve both spatially and kinematically with sub-kpc scale resolution.

\section{Sample and data}

We used near-IR data from four ULIRGs at $0.043 \leq z \leq 0.083$ obtained with the Spectrograph for Integral Field Observations in the Near Infrared (SINFONI) on the Very Large Telescope (VLT). These are IRAS 12112+0305, IRAS 14348-1447, IRAS 17208-0014, and IRAS 22491-1808. They are the four closest ULIRGs from a representative sample that was previously published by Piqueras López et al. (2012, hereafter PL12). Three of the four ULIRGs consist of two galaxies that are in a pre-coalescent stage of the merger, with the individual nuclei still clearly separated by at least several kpc. Therefore, our sample of four ULIRGs consists of seven (merging) galaxies.

The data were previously presented in PL12 and have a spaxel-size of $0.125^{\prime \prime}$, seeing of $\sim 0.6^{\prime \prime}$, and dispersion of $2.45 \AA \mathrm{pixel}^{-1}$. The spectral resolution is $6.4 \pm 0.6 \AA$, which corresponds to $90 \pm 8 \mathrm{~km} \mathrm{~s}^{-1}$ for the $\mathrm{H}_{2} 1-0 \mathrm{~S}(1)$ emission line at $2.1218 \mu \mathrm{m}$. PL12 fitted a single Gaussian to the spatially resolved $\mathrm{H}_{2} 1-0 \mathrm{~S}(1)$ line to retrieve the global kinematics of the molecular gas. However, in the four ULIRGs that we describe in this paper, the kinematic structure of the hot molecular gas appeared more complex than that. To more accurately derive the gas kinematics, we modified the data analysis routine of PL12 to fit a second Gaussian component to the $\mathrm{H}_{2}$ line profiles of these ULIRGs (Fig. 1). This routine was compiled for the Interactive Data Language (IDL) and uses the MPFIT package for the $\chi^{2}$ minimization (Markwardt 2009). We placed no constraints on the fitting parameters and visually inspected all fits to ensure their validity. For each component we created a flux, velocity, and dispersion map, which were boxcar-smoothed spatially by three pixels.

\section{Results}

Figures 2-5 show the results of our two-component Gaussian fit to the $\mathrm{H}_{2}$ 1-0 S(1) line in IRAS $12112+0305$, IRAS 143481447, IRAS 17208-0014, and IRAS 22491-1808. In all four ULIRGs, the Gaussian component with the highest peak flux is typically also the narrowest of the two components, with a full width at half the maximum intensity (FWHM) in the range of $\sim 180-280 \mathrm{~km} \mathrm{~s}^{-1}$. This component unambiguously traces the gas disk that was previously discussed in these ULIRGs by

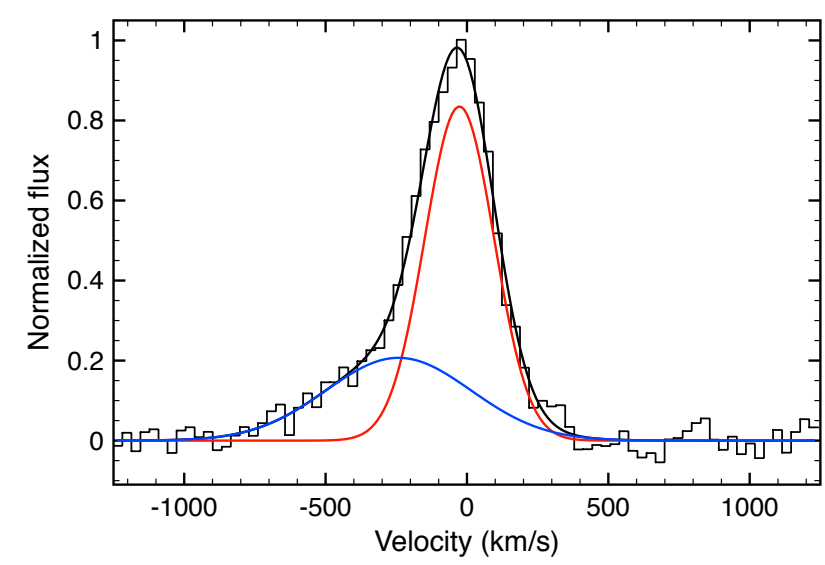

Fig. 1. Example of a two-component Gaussian fit to the spectrum of IRAS 14348-1447. Details of this spectrum, as well as the spectra of the other ULIRGs, are given in Fig. 6.

PL12. We will refer to this primary Gaussian component as the "narrow" component. The secondary Gaussian component reveals emission in the wings of the $\mathrm{H}_{2}$ line profile, and is typically broader and less luminous than the primary disk component. We therefore refer to this secondary Gaussian component as the "broad" component, with the caveat that in those spaxels where this secondary component is weak, it may appear narrower than the primary component. This broad-component emission reveals gas features that were not previously found by the single-component Gaussian approach of PL12. In Appendix A we describe the basic properties of the broad-component emission in each ULIRG.

The broad-component emission in the NE galaxy in the IRAS 12112+0305 pair (Fig. 2), the SW galaxy in the IRAS 14348-1447 pair (Fig. 3), and the E galaxy in the IRAS 22491-1808 pair (Fig. 5) is shown in more detail in Fig. 6. We find that in these systems the broad-component gas is decoupled from the narrow-component disk for two reasons: a) at the location of the most blueshifted emission in the maps of Fig. 6, the difference between the peak velocity of the broad and the narrow component in the emission-line profile exceeds the maximum rotational velocity of the narrow-component disk; b) the broad-component gas is blueshifted in the regions where the narrow-component disk is either redshifted or at the systemic velocity. We therefore argue that these three ULIRGs show an outflow of hot molecular $\mathrm{H}_{2}$ gas. The emission-line profiles of the $\mathrm{H}_{2}$ outflows stretch up to a maximum blueshifted velocity at zero intensity of $\sim 700 \mathrm{~km} \mathrm{~s}^{-1}$ for IRAS 12112+0305, $\sim 900 \mathrm{~km} \mathrm{~s}^{-1}$ for IRAS 14348-1447, and $\sim 500 \mathrm{~km} \mathrm{~s}^{-1}$ for IRAS 22491-1808 (Fig. 6). In Sect. 3.1, we will discuss in detail the geometry and mass of the outflows.

As a note of caution, in Appendix B we show that the velocity dispersion of the broad-component emission is significantly lower in IRAS 22491-1808 than it is in IRAS 12112+0305 and IRAS 14348-1447. Although the broad-component emission in IRAS 22491-1808 appears to be an outflow that is de-coupled from the slowly rotating narrow-component disk (Fig. 5), the relatively low velocities imply that future observations with JWST or the Atacama Large Millimeter/submillimeter Array (ALMA) are needed to completely rule out internal gas kinematics playing a role in producing the broad-component emission in IRAS 22491-1808. 
B. H. C. Emonts et al.: Outflows of hot molecular gas in ULIRGs
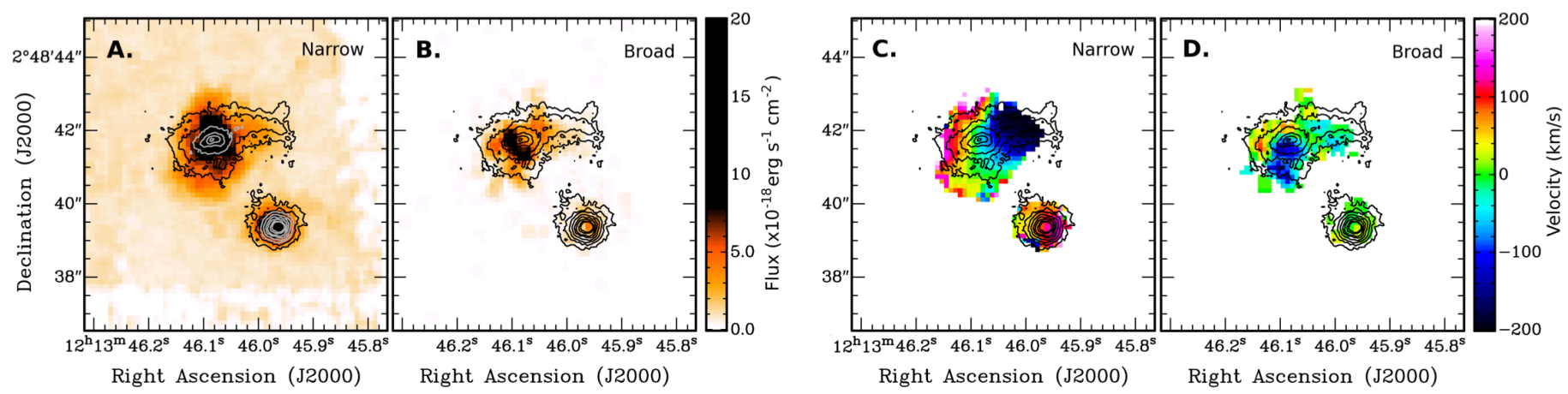

Fig. 2. Decomposition of the $\mathrm{H}_{2}$ 1-0 S(1) emission in IRAS 12112+0305. Left: flux of the narrow (A) and broad (B) component of the $\mathrm{H}_{2}$ emission. Right: velocity map of the narrow (C) and broad (D) component of the $\mathrm{H}_{2}$ emission in the region where the corresponding flux is $\geq 2 \times 10^{-18} \mathrm{erg} \mathrm{s}^{-1} \mathrm{~cm}^{-2}$ spaxel ${ }^{-1}$ for the narrow and $\geq 0.7 \times 10^{-18} \mathrm{erg} \mathrm{s}^{-1} \mathrm{~cm}^{-2}$ spaxel ${ }^{-1}$ for the broad component. The dark contours visualize an image taken with the Hubble Space Telescope Near Infrared Camera and Multi-Object Spectrometer (HST/NICMOS) in the combined $F 220 M+F 160 W+F 110 W$ filters (Scoville et al. 2000). HST contours start at $\sim 7 \sigma$, and are drawn at $12,19,28,47,71,95,143,190 \%$ of the peak intensity of the NE galaxy, which is the galaxy that shows the broad-component emission.
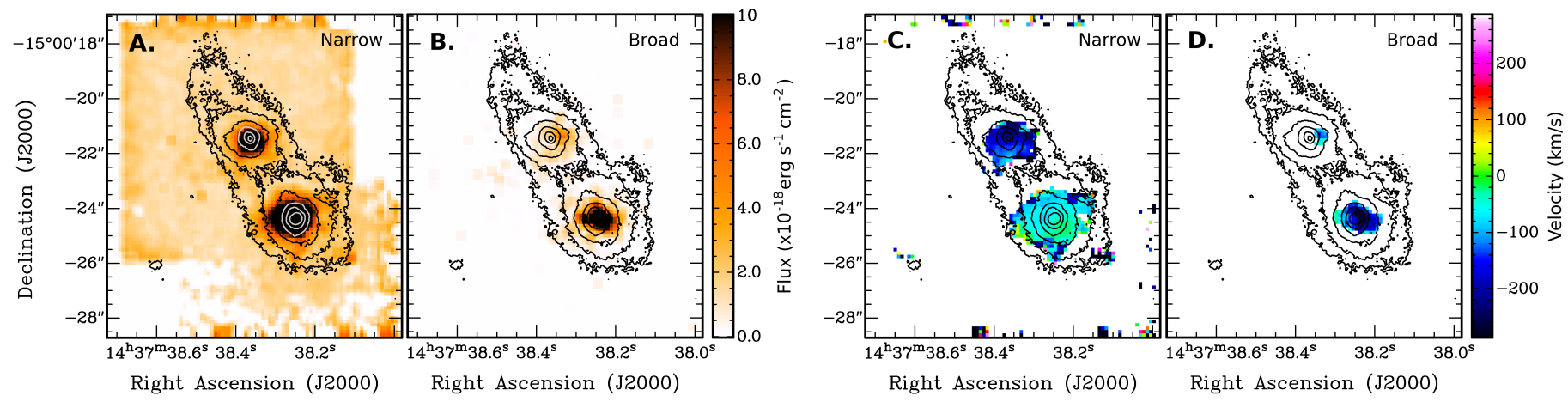

Fig. 3. Decomposition of the $\mathrm{H}_{2}$ 1-0 S(1) emission in IRAS 14348-1447. Left: flux of the narrow (A) and broad (B) component of the $\mathrm{H}_{2}$ emission. Right: velocity map of the narrow (C) and broad (D) component of the $\mathrm{H}_{2}$ emission in the region where the corresponding flux is $\geq 3 \times 10^{-18} \mathrm{erg} \mathrm{s}^{-1} \mathrm{~cm}^{-2}$ spaxel $^{-1}$ for both the narrow and the broad component. The dark contours visualize an image taken with HST/NICMOS in the combined $F 220 M+F 160 W+F 110 W$ filters (Scoville et al. 2000). HST contours start at $\sim 5 \sigma$, and are drawn at 1, 2, 5, 9, 18, 36\% of the peak intensity of the SW galaxy, which is the galaxy that shows the broad-component emission.
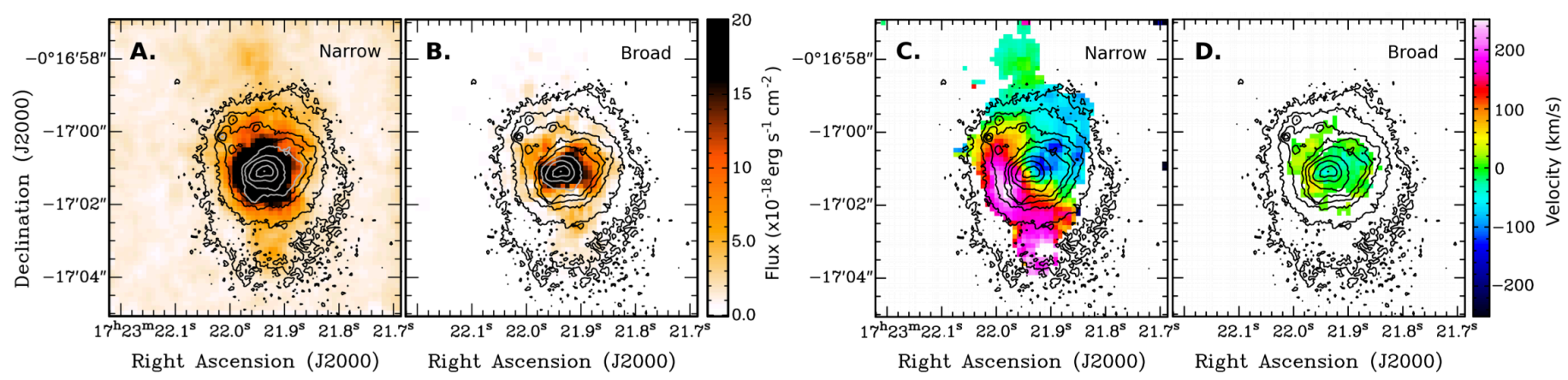

Fig. 4. Decomposition of the $\mathrm{H}_{2}$ 1-0 S(1) emission in IRAS 17208-0014. Left: flux of the narrow (A) and broad (B) component of the $\mathrm{H}_{2}$ emission. Right: velocity map of the narrow (C) and broad (D) component of the $\mathrm{H}_{2}$ emission in the region where the corresponding flux is $\geq 2.5 \times 10^{-18} \mathrm{erg} \mathrm{s}^{-1} \mathrm{~cm}^{-2}$ spaxel ${ }^{-1}$ for the narrow and $\geq 3.5 \times 10^{-18} \mathrm{erg} \mathrm{s}^{-1} \mathrm{~cm}^{-2}$ spaxel $^{-1}$ for the broad component. The dark contours visualize an image taken with HST/NICMOS in the combined $F 220 M+F 160 W+F 110 W$ filters (Scoville et al. 2000). HST contours start at $\sim 5 \sigma$, and are drawn at $2.5,5,10,15,20,30,50,75,100 \%$ of the peak intensity.

The fourth target in our sample, IRAS 17208-0014, shows broad-component emission that is clearly spatially resolved, but has velocities close to the systemic velocity of the galaxy. The nature of the broad-component emission in IRAS 17208-0014 is far less clear than it is in IRAS $12112+0305,14348-1447$, and 22491-1808. We will discuss IRAS 17208-0014 in detail in Sect. 3.2.

\subsection{Physical parameters of the $\mathrm{H}_{2}$ outflows}

The outflows that we detect in IRAS $12112+0305$, IRAS 14348 1447, and IRAS 22491-1808 (Fig. 6) are resolved both kinematically and spatially, which makes our SINFONI data ideal for further investigating the physical properties of these outflows. Table 1 shows the physical parameters that we derive from our 

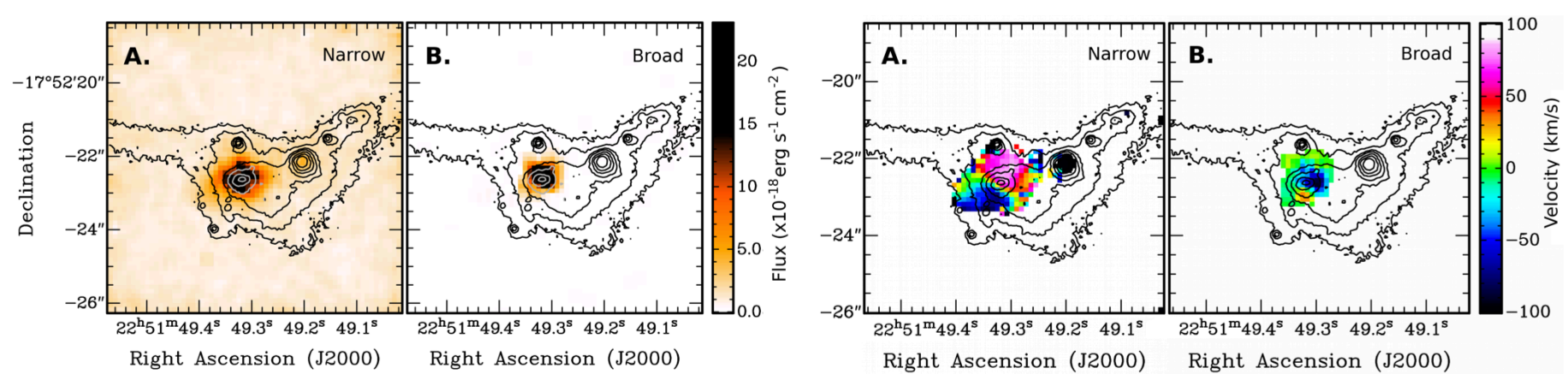

Fig. 5. Decomposition of the $\mathrm{H}_{2}$ 1-0 S(1) emission in IRAS 22491-1808. Left: flux of the narrow (A) and broad (B) component of the $\mathrm{H}_{2}$ emission. Right: velocity map of the narrow (C) and broad (D) component of the $\mathrm{H}_{2}$ emission in the region where the corresponding flux is $\geq 2.2 \times 10^{-18} \mathrm{erg} \mathrm{s}^{-1} \mathrm{~cm}^{-2}$ spaxel ${ }^{-1}$ for the narrow and $\geq 0.4 \times 10^{-18} \mathrm{erg} \mathrm{s}^{-1} \mathrm{~cm}^{-2}$ spaxel ${ }^{-1}$ for the broad component. The dark contours visualize an image taken with HST/NICMOS in the combined $F 220 M+F 160 W+F 110 W$ filters (Scoville et al. 2000). HST contours start at $\sim 5 \sigma$, and are drawn at $7,11,21,32,53,85,150 \%$ of the peak intensity of the E galaxy, which is the galaxy that shows the broad-component emission.

Table 1. Physical parameters of the $2.12 \mu \mathrm{m} \mathrm{H}_{2} 1-0 \mathrm{~S}(1)$ line features.

\begin{tabular}{lcccc}
\hline \hline & $12112+0305(\mathrm{NE})$ & $14348-1447(\mathrm{SW})$ & $22491-1808(\mathrm{E})$ & $17208-0014$ \\
\hline$z$ & 0.07310 & 0.08300 & 0.07776 & 0.04281 \\
$D_{\mathrm{L}}(\mathrm{Mpc})$ & 336 & 382 & 347 & 189 \\
$F_{\text {broad }}\left(\times 10^{-16} \mathrm{erg} \mathrm{s}^{-1} \mathrm{~cm}^{-2}\right)$ & $5.0 \pm 2.6$ & $6.4 \pm 1.5$ & $5.8 \pm 1.8$ & $22 \pm 8$ \\
$\Delta v\left(\mathrm{~km} \mathrm{~s}^{-1}\right)$ & $-190 \pm 65$ & $-215 \pm 55$ & $-160 \pm 75$ & - \\
$F W H M_{\text {narrow }}\left(\mathrm{km} \mathrm{s}^{-1}\right)^{\dagger}$ & $265 \pm 20$ & $275 \pm 15$ & $185 \pm 25$ & $255 \pm 20$ \\
$F W H M_{\text {broad }}\left(\mathrm{km} \mathrm{s}^{-1}\right)^{\dagger}$ & $465 \pm 50$ & $600 \pm 60$ & $310 \pm 75$ & $340 \pm 40$ \\
$v_{\text {max(broad) }}\left(\mathrm{km} \mathrm{s}^{-1}\right)$ & -430 & -520 & -320 & - \\
Size $_{\text {broad }}(\mathrm{kpc}$ & $1.6\left(1.0^{\prime \prime}\right)$ & $0.9\left(0.5^{\prime \prime}\right)$ & $0.7\left(0.4^{\prime \prime}\right)$ & $1.9\left(2.1^{\prime \prime}\right)$ \\
$M_{\left.\text {broad(hot } \mathrm{H}_{2}\right)}\left(M_{\odot}\right)$ & $(6.8 \pm 3.7) \times 10^{3}$ & $(8.4 \pm 2.2) \times 10^{3}$ & $(5.9 \pm 1.9) \times 10^{3}$ & $(7.5 \pm 2.7) \times 10^{3}$ \\
$\dot{M}_{\left.\text {broad(total } \mathrm{H}_{2}\right)}\left(M_{\odot} \mathrm{yr}^{-1}\right)^{\ddagger}$ & 30 & 85 & 45 & - \\
\hline
\end{tabular}

Notes. The redshift is indicated by $z, D_{\mathrm{L}}$ is the luminosity distance (PL12), $F_{\text {broad }}$ the flux of the broad component (see Sect. 3.1), $\Delta v=v_{\text {peak(broad) }}-$ $v_{\text {peak(narrow) }}$ the velocity difference between peak of the broad and the peak of the narrow component, FWHM the full width at half the maximum intensity of the components in the line-profile, $v_{\max (\mathrm{broad})}=\Delta v-(F W H M / 2)$ the maximum outflow velocity (e.g., Rupke et al. 2005; Arribas et al. 2014), $R_{\text {broad }}$ the total extent of the broad component, $M_{\left(\text {hot } \mathrm{H}_{2}\right)}$ the derived mass of the hot molecular gas (Sect. 3.1.2), and $\left.\dot{M}_{(\mathrm{total}} \mathrm{H}_{2}\right)$ the estimated mass outflow rate of the total (hot + cold) molecular gas, assuming a hot-to-cold gas mass ratio of $6 \times 10^{-5}$ (Sect. 3.1.2). The measurements of $F_{\text {broad }}$ and $R_{\text {broad }}$ are derived from the flux and velocity maps of Figs. 2-5, while $\Delta v$, FWHM, and $v_{\max (\text { broad) }}$ are derived from the $\mathrm{H}_{2}$ line profiles shown in Fig. 6. For additional properties of the narrow-component emission, we refer to PL12 and Appendix A. ${ }^{(\dagger)}$ Corrected for instrumental broadening of $90 \mathrm{~km} \mathrm{~s}^{-1}$ (PL12). ${ }^{(\ddagger)}$ Based on a hot-to-cold molecular gas mass ratio of $6 \times 10^{-5}$ (Emonts et al. 2014; Pereira-Santaella et al. 2016).

measurements. Errors in the Gaussian fitting procedure, as well as the redshift determination, can affect the measurement of the integrated flux of the broad component $F_{\text {broad }}$ and associated mass of the outflow. This is particularly true when the velocity difference between the broad and narrow component is small. Therefore, simply summing up the flux of the broad-component in Figs. 2, 3, and 5 likely provides a firm upper limit on the total flux of the outflows. A conservative lower limit is given by summing the broad-component flux only in those spaxels where the broad and narrow component are clearly separated kinematically. For this, we only take into account the total broadcomponent line emission in those spaxels where the difference in central peak velocity between the broad and narrow component, as shown in Figs. 2, 3, and 5, is $|\Delta v| \geq 100 \mathrm{~km} \mathrm{~s}^{-1}$. In Table 1, $F_{\text {broad }}$ is the average value between the upper- and the lowerlimit calculation, with corresponding uncertainties reflecting the difference between the two approaches.

The temperature of the $\mathrm{H}_{2}$ gas in the outflows can in principle be estimated if multiple rovibrational $\mathrm{H}_{2}$ lines are measured (e.g., Davies et al. 2003). In the case of IRAS 12112+0305, IRAS 14348-1447, and IRAS 22491-1808 we cannot determine the $\mathrm{H}_{2}$ temperature from our SINFONI data, because the $\mathrm{H}_{2}$ $1-0 \mathrm{~S}(0)\left(\lambda_{\text {rest }}=2.22 \mu\right)$ and $2-1 \mathrm{~S}(1)\left(\lambda_{\text {rest }}=2.25 \mu \mathrm{m}\right)$ lines are faint and fall in a region of the spectrum that is dominated by strong sky lines. This makes it impossible to derive an accurate flux of the broad-component emission from $\mathrm{H}_{2} 1-0 \mathrm{~S}(0)$ and $\mathrm{H}_{2}$ 2-1 S(1).

\subsubsection{Geometry and kinematics of the outflows}

The measured size of the outflow region in IRAS $12112+0305$, IRAS 14348-1447, and IRAS 22491-1808 ranges from 0.7$1.6 \mathrm{kpc}$. These are strict lower limits, given that we do not know, and thus do not correct for, the inclination of the outflow. The outflows do not extend across the entire area of the narrowcomponent disk. In fact, their morphologies suggest that the outflows originate from the nuclear region (see Appendix A for details).

Figure 6 suggests that the outflows in IRAS $12112+0305$ and IRAS 22491-1808 appear to be aligned somewhat closer to the minor than to the major axis of the galaxies, although not to such an extent that we draw any firm conclusions from this. For IRAS 14348-1447, the galaxy appears to be oriented close to face-on, while the high radial velocity and small spatial extent of the outflow likely indicate that the gas is flowing mostly along our line of sight. For all three ULIRGs, the outflow velocity reaches a maximum at roughly $0.5 \mathrm{kpc}$ distance from the core. These properties are similar to those of the conical, nuclear 

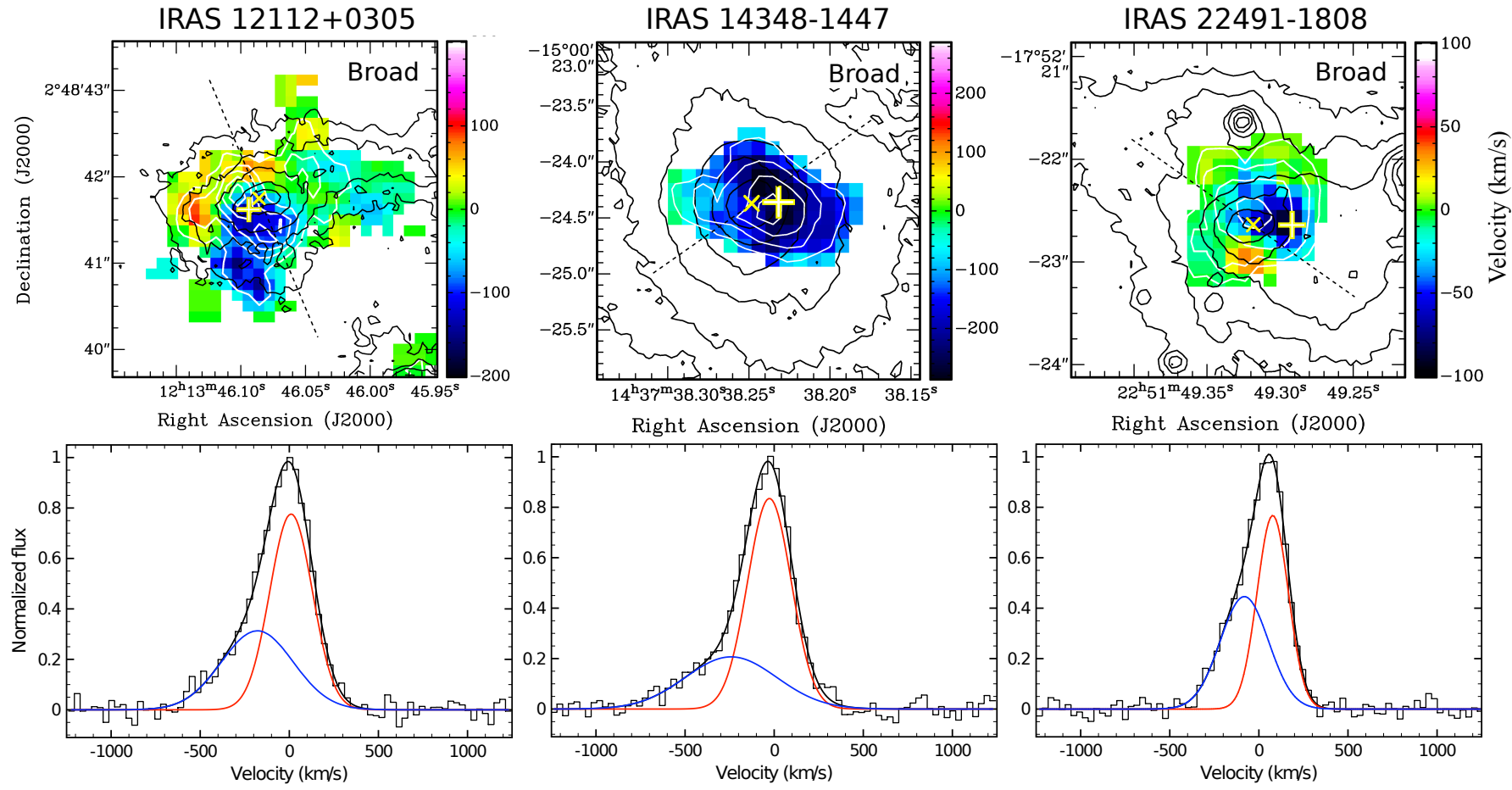

Fig. 6. Top: zoom-in of the $\mathrm{H}_{2}$ outflows from Figs. 2, 3, and 5. The $\mathrm{H}_{2}$ velocity map of the broad-component emission is shown in color, with overlaid in white contours the $\mathrm{H}_{2}$ total-intensity map of the broad component and in black contours the HST/NICMOS image from Figs. 2, 3, and 5. Contour levels of the total-intensity maps of the broad component are: 2, 4, 6, 8, 16 $\times 10^{-18} \mathrm{erg} \mathrm{s}^{-1} \mathrm{~cm}^{-2}$ spaxel $^{-1}$ (IRAS 12112+0305); $5,9,13,17 \times 10^{-18} \mathrm{erg} \mathrm{s}^{-1} \mathrm{~cm}^{-2}$ spaxel ${ }^{-1}$ (IRAS 14348-1447); 1, 3, 9, $27 \times 10^{-18} \mathrm{erg} \mathrm{s}^{-1} \mathrm{~cm}^{-2}$ spaxel $^{-1}$ (IRAS 22491-1808). The small cross ( $\times$ ) marks the peak of the IR-emission in the NICMOS image, which is presumably the center of the galaxy. The large cross $(+)$ marks the location where the outflow reaches a maximum velocity at zero intensity, and against which we extracted the $\mathrm{H}_{2}$ spectra shown below. The dashed line represents the direction of the minor axis, as we estimated from the kinematic of the narrow-component disk and the HST/NICMOS imaging (see Appendix A). Bottom: $\mathrm{H}_{2} 1-0 \mathrm{~S}(1)$ spectra extracted from a region of five pixels (three pixels both $\mathrm{E}$ to $\mathrm{W}$ and $\mathrm{N}$ to $\mathrm{S}$, as marked with the "+" sign above). The black line shows a double Gaussian fit, consisting of a narrow (red) and broad (blue) component.

$\mathrm{H}_{2}$ outflow that was first imaged with near-IR integral-field spectroscopy in QSO F08572+3915 (Rupke \& Veilleux 2013b).

The velocity FWHM of the broad-component emission in the outflows is roughly twice that of the narrow-component emission in the disk. In part, this may be related to a backflow of gas, or to the fact that the opening angle of the outflowing gas causes a superposition of radial velocities along our line of sight. However, in particular in the case of IRAS 12112+0305, the conical outflow appears to have a modest opening angle of $\sim 40^{\circ}$ (Appendix A). It is therefore more likely that the turbulence of the $\mathrm{H}_{2}$ gas in the outflow is higher than that in the narrow-component disk. With a $2 \times$ larger FWHM, the turbulent kinetic energy of the gas in the outflow, $E_{\mathrm{kin}}^{\mathrm{turb}}=\frac{3}{2} \cdot M \sigma^{2}$ $(\sigma=F W H M / 2.35)$, is up to a factor of four higher than that of the gas in the disk.

\subsection{2. $\mathrm{H}_{2}$ mass of the outflows}

To estimate the mass of the hot molecular gas that is locked up in the broad-component emission, we follow Scoville et al. (1982) and Mazzalay et al. (2013):

$M_{\mathrm{H}_{2}} \simeq 5.1 \times 10^{13}\left(\frac{D_{\mathrm{L}}}{\mathrm{Mpc}}\right)^{2}\left(\frac{F_{1-0 \mathrm{~S}(1)}}{\mathrm{erg} \mathrm{s}^{-1} \mathrm{~cm}^{-2}}\right) 10^{\left(0.4 \cdot A_{2.2}\right)}$,

where $M_{\mathrm{H}_{2}}$ is the hot molecular $\mathrm{H}_{2}$ mass in $M_{\odot}, D_{\mathrm{L}}$ is the luminosity distance to the galaxy, and $A_{2.2 \mu \mathrm{m}}$ is the extinction at $2.2 \mu \mathrm{m}$. In this case, we substitute $F_{1-0 \mathrm{~S}(1)}$ for the flux of the broad component ( $F_{\text {broad }}$ in Table 1$)$. This approach assumes thermalized gas conditions and $T=2000 \mathrm{~K}$, with a population fraction in the $(v, J)=(1,3)$ level of $f_{(1,3)}=0.0122$. If a fraction of the $\mathrm{H}_{2}$ gas was sub-thermally excited, $f_{1,3}$ would be lower and Eq. (1) would thus give a lower limit to the $\mathrm{H}_{2}$ mass. Following Piqueras López et al. (2013), $A_{2.2 \mu \mathrm{m}} \approx 0.1 \times A_{\mathrm{V}}$, with $A_{\mathrm{V}}$ the visual extinction, based on the extinction law described by Calzetti et al. (2000; see also, e.g., Rieke \& Lebofsky 1985; Fitzpatrick 1999; Indebetouw et al. 2005). Because the broadcomponent $\mathrm{H}_{2}$ emission is resolved on scales of a few $\mathrm{kpc}$, we use $A_{\mathrm{V}, R(\mathrm{eff})}$ from Piqueras López et al. (2013), which is the visual extinction within the effective radius of the $\mathrm{H} \alpha$ emission (Arribas et al. 2012). We note, however, that this implicitly assumes that the extinction in the outflow is similar to that inferred from the overall (narrow + broad component) $\mathrm{H}_{2}$ gas in this region.

Based on Eq. (1), the broad-component emission in IRAS 12112+0305, IRAS 14348-1447, and IRAS 22491-1808 represents outflows of hot molecular gas with masses of $M_{\mathrm{H} 2 \text { (hot) }} \sim 6-8 \times 10^{3} M_{\odot}$ (Table 1). The total mass of the outflowing molecular gas consists of a hot and cold component. Recently, we derived hot-to-cold molecular gas mass ratios of $6-7 \times 10^{-5}$ for $\mathrm{H}_{2}$ outflows in two nearby luminous infrared galaxies (LIRGs; $10^{11} \leq L_{\mathrm{IR}}<10^{12} L_{\odot}$ ), by comparing SINFONI and ALMA data (Emonts et al. 2014; Pereira-Santaella et al. 2016). The most prominent of these two outflows, in the LIRG NGC 3256, has a hot molecular gas mass of $\sim 1200 M_{\odot}$, which is roughly a factor of six lower than what we observe in our ULIRGs. If we assume a similar hot-to-cold molecular gas mass ratio of $6 \times 10^{-5}$, then the total molecular gas masses involved in the ULIRG outflows are of the order of $M_{\mathrm{H} 2} \sim 1 \times 10^{8} M_{\odot}$. This 

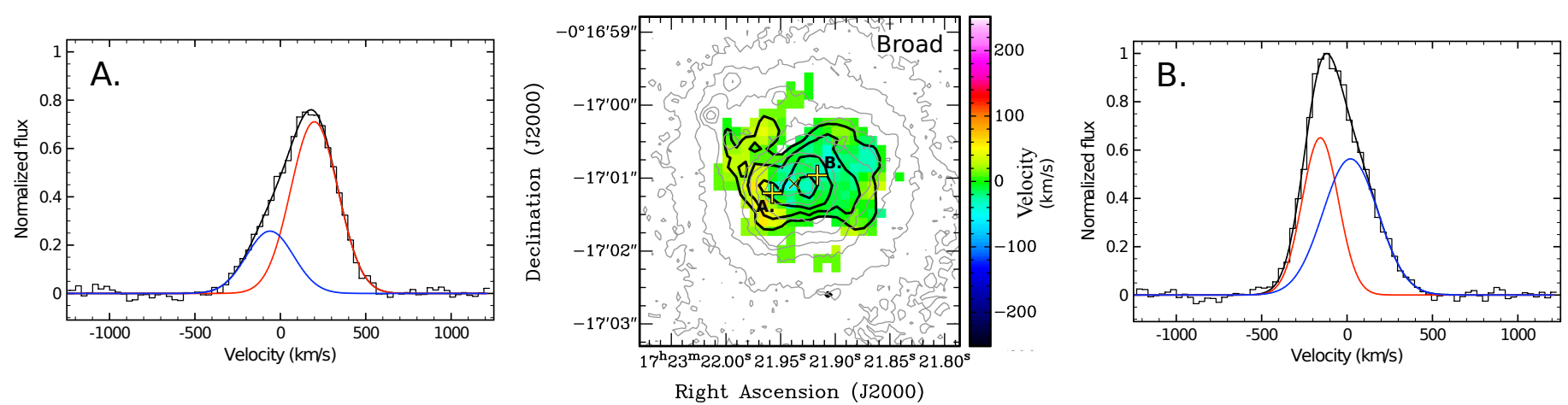

Fig. 7. Middle: zoom-in of the broad-component $\mathrm{H}_{2}$ emission in IRAS 17208 from Fig. 4. The $\mathrm{H}_{2}$ velocity map of the broad-component emission is shown in color, with overlaid in thick black contours the $\mathrm{H}_{2}$ total-intensity map of the broad component and in thin gray contours the HST/NICMOS image from Fig. 4. Contour levels of the total-intensity maps of the broad component are: $6,12,24,48 \times 10^{-18} \mathrm{erg} \mathrm{s}^{-1} \mathrm{~cm}^{-2} \mathrm{spaxel}^{-1}$. The small yellow/black cross $(\times)$ marks the center of the galaxy from the HST/NICMOS image, while the large black/yellow crosses $(+)$ mark the location against which we extracted the $\mathrm{H}_{2}$ spectra A and B. Left/right: $\mathrm{H}_{2} 1-0 \mathrm{~S}(1)$ spectra extracted from a region of five pixels (three pixels both E to W and $\mathrm{N}$ to $\mathrm{S}$, as marked with the "+" signs in the middle panel). The black line shows a double Gaussian fit, consisting of a narrow (red) and broad (blue) component.

corresponds to mass outflow rates of $\dot{M}_{\mathrm{H}_{2}}=\frac{M_{\mathrm{H} 2(\text { total) }} \cdot v_{\max } \sin (i)}{R_{\text {outflow }} \cos (i)} \approx$ 30-85 $\tan (i) M_{\odot} \mathrm{yr}^{-1}$, where $R_{\text {outflow }}$ is the size of the outflow region, $v_{\max }$ the maximum outflow velocity (Table 1 ), and $i$ the line-of-sight inclination ${ }^{1}$. Upcoming $\mathrm{CO}$ observations in the millimeter regime will be able to confirm these estimates.

\subsection{IRAS 17208-0014}

IRAS 17208-0014 shows broad-component emission that is clearly spatially resolved, but shows little kinematic structure. The overall $\mathrm{H}_{2}$ kinematics in IRAS 17208-0014 appear to follow the $\mathrm{CO}(2-1)$ kinematics of cold molecular gas discussed in detail by García-Burillo et al. (2015). We argue that it is unlikely that the broad-component $\mathrm{H}_{2}$ emission represents an outflow similar to what we see in IRAS $12112+0305$, IRAS 14348-1447, and IRAS 22491-1808. The reason is that the broad-component gas in IRAS 17208-0014 has a velocity that is close to the systemic velocity across the narrowcomponent disk. Moreover, the broad-component emission is spread across a large fraction of the narrow-component disk. Therefore, if this broad-component emission represents an outflow, it would have to be gentle and widespread, reminiscent of a galactic wind with very low radial velocity. It is interesting to note that a widespread outflow of neutral gas has been reported in $\mathrm{NaD}$ absorption across several kpc (Martin 2006; Rupke \& Veilleux 2013a). On the contrary, there is no observational evidence for a similar widespread outflow in the ionized gas phase (e.g., Arribas \& Colina 2003; Westmoquette et al. 2012; Rupke \& Veilleux 2013a, see Appendix A.3).

However, it is good to keep in mind that the broadcomponent emission is separated from the narrow-component emission as a result of our Gaussian fitting procedure. In fact, in the central kpc-scale region, the morphology of the broadcomponent emission seems to resemble the structure seen with the Near Infrared Camera and Multi-Object Spectrometer (NICMOS) on the Hubble Space Telescope (HST), while fainter broad-component emission further out shows tentative indications for a spiral-like morphology (Figs. 4 and 7). It is therefore possible that the broad-component emission is merely a low-velocity wing to the narrow-component gas, perhaps

\footnotetext{
1 Because we do not know the inclination of these systems, we will ignore the inclination effects in the remainder of this paper.
}

representing internal gas dynamics or gas falling into the center of the galaxy. Alternatively, the broad-component $\mathrm{H}_{2}$ emission could present low-velocity gas outside the galaxy disk, which was previously deposited through a galactic-scale outflow and possibly mixed with low-angular-momentum gas from the halo (see, e.g., Fraternali \& Binney 2006). Similar low-velocity gas has been seen outside the disks of nearby galaxies in neutral hydrogen gas (e.g., Boomsma et al. 2005; Oosterloo et al. 2007), and bears resemblance to the low-velocity $\mathrm{NaD}$ component seen in the LIRG IRAS F11506-3851 by Cazzoli et al. (2014). Finally, Medling et al. (2014) argue that the core of IRAS 172080014 consists of two close nuclei separated by $\sim 200 \mathrm{pc}$. It is interesting to speculate that the narrow- and broad-component emission result from two superposed gas disks. Based on Fig. 4, the dominant narrow-component disk would be substantially inclined. On the contrary, the lack of line-of-sight kinematics among the broad-component emission indicates that this would represent a face-on disk with a size of at least $\sim 2 \mathrm{kpc}$ and possible spiral-type morphology. While the nature of the broadcomponent emission in IRAS 17208-0014 remains unclear, detailed observations with ALMA or JWST will be able to discern between the different scenarios.

\subsection{Upper limit on the $\mathrm{H}_{2}$ outflow in IRAS 17208-0014}

García-Burillo et al. (2015) also discovered a faint, blueshifted $\mathrm{CO}(2-1)$ outflow in IRAS $17208-0014$. Figure 8 shows the $\mathrm{H}_{2} 1-$ $0 \mathrm{~S}(1)$ spectrum extracted across a circular aperture with an area of 13 spaxels at the location where the $\mathrm{CO}$ outflow was found. We do not detect an $\mathrm{H}_{2}$ counterpart to this $\mathrm{CO}$ outflow in our SINFONI data. Figure 8 shows that any potential outflow is buried well within the noise, and affected by features within the near-IR continuum. The $\mathrm{CO}$ outflow stretches roughly from -500 to $-700 \mathrm{~km} \mathrm{~s}^{-1}$, which is outside the line profile of the narrow-component $\mathrm{H}_{2} 1-0 \mathrm{~S}(1)$ emission that we observe with SINFONI (Fig. 7). Our SINFONI data show an rms noise level of $\sim 8 \times 10^{-16} \mathrm{erg} \mathrm{s}^{-1} \mathrm{~cm}^{-2} \mu \mathrm{m}^{-1}$ per $0.125^{\prime \prime}$ spaxel and per $2.45 \AA\left(35 \mathrm{~km} \mathrm{~s}^{-1}\right)$ resolution element in the dispersion direction. We set a conservative upper limit on the integrated line flux of a spatially unresolved $\mathrm{H}_{2} 1-0 \mathrm{~S}(1)$ outflow across $200 \mathrm{~km} \mathrm{~s}^{-1}$ by assuming a $10 \sigma$ detection when binning the data across $200 \mathrm{~km} \mathrm{~s}^{-1}$ (six pixels) in dispersion direction and across the 


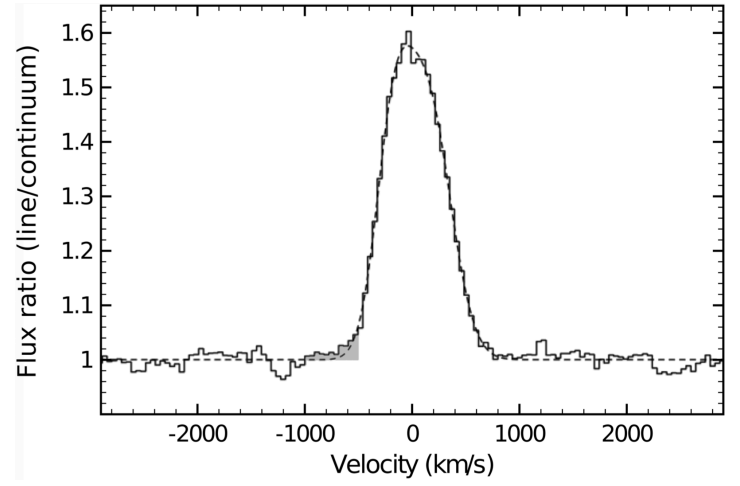

Fig. 8. Spectrum of the $\mathrm{H}_{2} 2.1218 \mu \mathrm{m}$ line in IRAS 17208-0014 centered one spaxel $\left(0.125^{\prime \prime}\right)$ south of the core, where García-Burillo et al (2015) found an outflow in $\mathrm{CO}(2-1)$. The spectrum was extracted against a circular aperture with an area of 13 pixels, and therefore includes the strong nuclear near-IR continuum emission. Plotted on the $y$-axis is the ratio of the emission-line flux and the continuum flux. The dashed line shows the best-fit model of the narrow-component disk. No convincing outflow is seen in $\mathrm{H}_{2}$. The emission in the gray shaded area, which stretches from -1000 to $-500 \mathrm{~km} \mathrm{~s}^{-1}$ and is affected by noise and continuum features, comprises an $\mathrm{H}_{2}$ mass of $\sim 110 M_{\odot}$. This is a factor of four below the firm upper limit that we derive for an $\mathrm{H}_{2}$ outflow in IRAS 17208-0014 (see text for details).

$0.6^{\prime \prime}$ (18 pixels) seeing-disk in spatial directions. This is equivalent to $1 \sigma$ per raw pixel in the unbinned data for a signal distributed across the seeing-disk and across $200 \mathrm{~km} \mathrm{~s}^{-1}$. Following Sect. 3.1.2, this results in an upper limit on the outflow in hot molecular $\mathrm{H}_{2}$ gas of $M_{\mathrm{H} 2-\text { hot }} \lesssim 400 M_{\odot}$. For comparison, the $\mathrm{H}_{2}$ emission from the shaded region in Fig. 8 only comprises $M_{\mathrm{H} 2-\text { hot }} \lesssim 110 M_{\odot}$. Given the underlying near-IR continuum emission, we therefore argue that $M_{\mathrm{H} 2-\text { hot }} \lesssim 400 M_{\odot}$ is a conservative upper limit.

With our assumed hot-to-cold molecular gas mass ratio of $6 \times 10^{-5}$ (Sect. 3.1.2), the corresponding upper mass limit on the total (hot + cold) molecular outflow is $M_{\text {outflow }} \lesssim 6.7 \times 10^{6} M_{\odot}$, which is about a factor of seven lower than the CO outflow of $M_{\text {outflow }} \sim 4.6 \times 10^{7} M_{\odot}$ observed by García-Burillo et al. (2015). To bring both observations into agreement, we would have to put an upper limit on the hot-to-cold molecular gas mass ratio of $\lesssim 9 \times 10^{-6}$. This is substantially lower than the hot-to-cold molecular gas mass ratios of $6-7 \times 10^{-5}$ measured directly in the outflows of two nearby LIRGs with SINFONI and ALMA (Emonts et al. 2014; Pereira-Santaella et al. 2016). Nevertheless, it is within the $10^{-7}-10^{-5}$ range of generic values previously found by Dale et al. (2005) in samples of starburst galaxies and AGN. Therefore, our SINFONI data are not sensitive enough to either verify or rule out the existence of a hot $\mathrm{H}_{2}$ counterpart to the CO outflow found by García-Burillo et al. (2015).

\section{Discussion}

We discovered massive outflows of hot molecular $\mathrm{H}_{2}$ gas from three of four nearby ULIRGs (75\%), namely IRAS 12112+0305 (NE), IRAS 14348-1447 (SW), and IRAS 22491-1808 (E). These three systems are all in a pre-coalescent stage, and for all three we detect an outflow in only one of the two merging galaxies. We therefore detect a hot molecular outflow in three of the seven individual galaxies in our sample (43\%). However, we stress that our SINFONI data are only sensitive to tracing molecular gas outflows of $M_{\mathrm{H} 2 \text { (hot+cold) }} \sim 10^{8} M_{\odot}$ with outflow velocities $v_{\max } \sim$ several
$100 \mathrm{~km} \mathrm{~s}^{-1}$ (Table 1). For example, we lack the sensitivity to trace the hot $\mathrm{H}_{2}$ counterpart to the molecular outflow that was previously discovered in CO in the fourth ULIRG in our sample, IRAS 17208-0014 (García-Burillo et al. 2015, see Sect. 3.3). Sensitive near-IR integral-field spectroscopy of larger samples of ULIRGs is needed to derive meaningful conclusions about the detection rate of hot molecular $\mathrm{H}_{2}$ outflows in ULIRGs.

\subsection{Link to starburst or AGN activity}

IRAS 12112+0305, IRAS 14348-1447, IRAS 17208-0014, and IRAS 22491-1808 all have a prominent starburst component. Their nuclei are all radio-quiet, and have been classified as either low ionization nuclear emission-line regions (LINERs) or starburst/H II regions (e.g., Veilleux et al. 1999; Franceschini et al. 2003; Nardini et al. 2010). From high-resolution IR spectroscopy, Alonso-Herrero et al. (2016) show that IRAS 143481447 and IRAS 17208-0014 contain a faint AGN at mid-IR wavelengths.

However, X-ray observations classify the SW nucleus of IRAS 14348-1447 as a Compton-thick AGN candidate (Iwasawa et al. 2011). Previous work revealed that ionized and molecular gas outflows are on average faster and more massive in the presence of an AGN (Spoon et al. 2013; Arribas et al. 2014; Cicone et al. 2014; Hill \& Zakamska 2014; see also models by Narayanan et al. 2008). The fact that IRAS 14348-1447 (SW) shows the fastest mass outflow among our sample sources is in agreement with this previous work, and suggests that the $\mathrm{H}_{2}$ outflow in IRAS $14348-1447$ is driven by the Compton-thick AGN.

The energetics in IRAS 14348-1447 are also consistent with this scenario. Iwasawa et al. (2011) estimate that the X-ray luminosity of the SW galaxy of IRAS $14348-1447$ is $L_{\mathrm{X}(0.5-10 \mathrm{keV})} \sim$ $5.4 \times 10^{41} \mathrm{erg} \mathrm{s}^{-1}$. Following Elvis et al. (1994) and Ho (2008), the expected bolometric output of the AGN is roughly $20 \times$ $L_{\mathrm{X}(0.5-10 \mathrm{keV})}$, or $L_{\text {bol }} \times 1.1 \times 10^{43} \mathrm{erg} \mathrm{s}^{-1}$. The $\mathrm{H}_{2}$ outflow has a bulk flow of $\sim 200 \mathrm{~km} \mathrm{~s}^{-1}$, which means that it has taken roughly $5 \times 10^{6} \mathrm{yr}$ for the flow to reach $\sim 1 \mathrm{kpc}$ distance (Table 1 ). In $5 \times 10^{6} \mathrm{yr}$, the estimated energy output of the Compton thick $\mathrm{AGN}$ is $E_{\mathrm{AGN}} \sim 1.7 \times 10^{57} \mathrm{erg}$. This is likely a lower limit, given that the $0.5-10 \mathrm{keV}$ emission could be heavily absorbed. The total (bulk + turbulent) kinetic energy of the outflow is $E_{\text {tot }}=$ $E_{\mathrm{kin}}^{\text {turb }}+E_{\text {kin }}^{\text {bulk }}=\frac{3}{2} \cdot M \sigma^{2}+\frac{1}{2} \cdot M\left[v / \sin \left(i_{\text {outf }}\right)\right]^{2}=3.4 \pm 0.9 \times 10^{56} \mathrm{erg}$ (following Table 1). This is a strict lower limit, given that the inclination $i_{\text {outfl }}$ is likely to be large. Nevertheless, the energy output of the Compton-thick AGN appears to be sufficient to drive the outflow in IRAS 14348-1447.

\subsection{Effect on galaxy evolution}

Based on spectral energy distributions from the ultra-violet to far-infrared, da Cunha et al. (2010) derive stellar masses of $2.2,13.5$, and $4.2 \times 10^{10} M_{\odot}$ for IRAS $12112+0305$, IRAS 17208-0014, and IRAS 22491-1808, respectively. Assuming that this stellar mass is distributed equally between the merging galaxies in each ULIRG, the escape velocity at $2 \mathrm{kpc}$ distance from the galaxy ranges from $\sim 220 \mathrm{~km} \mathrm{~s}^{-1}$ for IRAS $12112+0305$ (NE) to $\sim 300 \mathrm{~km} \mathrm{~s}^{-1}$ for IRAS 224911808 (E). Arribas et al. (2014) instead derive dynamical masses of $4.6,7.4,2.1$, and $3.4 \times 10^{10} M_{\odot}$ for IRAS $12112+0305$, IRAS 14348-1447, IRAS 17208-0014, and IRAS 22491-1808, respectively. Following Eq. (7) of Arribas et al. (2014), assuming $r=2 \mathrm{kpc}$ and an isothermal sphere with truncation radio 
$r_{\text {max }}=20 \mathrm{kpc}$, this results in $v_{\mathrm{esc}} \sim 465,590$, and 400 for the merging ULIRGs IRAS 12112+0305, 14348, and 22491-1808, respectively. Therefore, while a small fraction of the outflowing molecular gas may escape the ULIRGs, the bulk of the outflowing molecular gas in IRAS 12112-0305 and IRAS 14348-1447, and possibly all of it in IRAS 22491-1808, will remain bound to the merging system. The outflows that we observe will therefore mainly re-distribute the gas within the ULIRGs, which may contribute to the dynamical processes that delay the formation of stars. However, the molecular gas itself will remain available within the system to fuel future star formation.

Moreover, Evans et al. (2002) and García-Marín et al. (2009) derive star formation rates of the order of 200-400 $M_{\odot} \mathrm{yr}^{-1}$ for IRAS 12112-0305 and IRAS 14348-1447, based on infrared, radio, and $\mathrm{H} \alpha$ observations. Our estimated molecular outflow rates of $\dot{M}_{\mathrm{H}_{2} \text { (total) }} \sim 30-85 M_{\odot} \mathrm{yr}^{-1}$ are at least a factor of a few lower than the star formation rates. This suggests that the effects that the molecular outflow have on delaying star formation are rather modest.

\subsection{Multi-phase gas outflows}

Multi-phase gas outflows, down to the lowest temperatures of molecular gas, can be an important aspect in the evolution of ULIRGs. A demonstration of this are the detailed studies done on the nearby quasar-type ULIRG MRK 231 (e.g., Rupke \& Veilleux 2011; Aalto et al. 2015; Alatalo 2015; Feruglio et al. 2015; Morganti et al. 2016; Veilleux et al. 2016). Such multi-phase gas outflows are likely to have cold, dense clouds entrained and accelerated by a flow of warm and hot gas (e.g., Wagner et al. 2012; Zubovas \& King 2014). A clumpy outflow of cold molecular gas was recently observed with ALMA in the LIRG IRAS F11506-3851, where CO-emitting clouds with sizes between 60 and $150 \mathrm{pc}$ are part of a multi-phase outflow (Pereira-Santaella et al. 2016).

The best studied phase of outflows is the ionized gas phase, as traced by optical emission lines. While a detailed discussion of ionized outflows is beyond the scope of this paper, it is interesting to note that among a large sample of nearby LIRGs and ULIRGs, Arribas et al. (2014) found that outflows are nearly ubiquitous in (U)LIRGs. The outflow velocities that they observe are similar to what we detect in $\mathrm{H}_{2}$ in our small sample of ULIRGs, while also the ionized gas outflows do not reach the escape velocity in all but the least massive systems (Arribas et al. 2014).

Considering the molecular gas, the nearby LIRG NGC 3256 was the first galaxy where we imaged a bi-conical molecular outflow both in the hot and cold molecular phase at $\sim 100$ pc resolution (Emonts et al. 2014, see also Sakamoto et al. 2014). The hot and cold components share a similar morphology and kinematics, which suggests that the hot and cold phase trace the same gas features. In NGC 3256 we derived a hot-to-cold molecular gas mass ratio of $\sim 6 \times 10^{-5}$, which is similar to that found in another nearby LIRG (Pereira-Santaella et al. 2016). The total molecular mass outflow rate of at least $\dot{M}_{\mathrm{H}_{2}} \gtrsim 20 M_{\odot} \mathrm{yr}^{-1}$ in the LIRG NGC 3256 is similar to that of the $\mathrm{H}_{2}$ outflows that we identified in the ULIRGs studied in this paper.

On the other hand, Cicone et al. (2014) studied molecular outflows through observations of $\mathrm{CO}$ in a sample of nearby ULIRGs. They find the presence of molecular outflows with mass outflow rates of several $100 M_{\odot} \mathrm{yr}^{-1}$, which are similar or larger than the star formation rates in these systems. For the ULIRGs that we study in this paper, we derive outflow rates of molecular gas that are a factor of a few lower than the star formation rates (Sect. 4.1). Therefore, our $\mathrm{H}_{2}$ results appear at odds with the CO results from Cicone et al. (2014). Of course, the $\mathrm{H}_{2} 2.12 \mu \mathrm{m}$ line only traces a small fraction of the total molecular gas mass, and its strength is subject to the local gas temperature and excitation (see, e.g., Rigopoulou et al. 2002). Complementary $\mathrm{CO}$ or mid-IR $\mathrm{H}_{2}$ observations of our sample sources are needed to further investigate the hot-to-cold molecular gas mass ratio.

Some of the most spectacular molecular gas outflows in ULIRGs have been detected with the Herschel Space Observatory in the $\mathrm{OH}$ line (e.g., Sturm et al. 2011; Spoon et al. 2013; Veilleux et al. 2013; González-Alfonso et al. 2017). The outflow velocities that we observe in the $\mathrm{H}_{2}$ line with SINFONI are within the range of the $\mathrm{OH}$ outflows detected with Herschel. However, the mass outflow rates derived from $\mathrm{OH}$ measurements by Sturm et al. (2011) and González-Alfonso et al. (2017) are nearly ubiquitously $\dot{M}_{\mathrm{H}_{2}} \gtrsim 100 M_{\odot} \mathrm{yr}^{-1}$. Our SINFONI data suggest also here that either molecular outflow rates in nearby ULIRGs are much more moderate than predicted based on $\mathrm{OH}$ studies, or that our assumed hot-to-cold gas mass ratio for $\mathrm{H}_{2}$ outflows in ULIRGs is too large. Alternatively, the $\mathrm{OH}$ studies may trace faster outflows that originate closer to the galaxy core, with higher $v_{\max }$ and smaller $R_{\text {outflow }}$. González-Alfonso et al. (2017) indeed found evidence for higher maximum outflow velocities seen in $\mathrm{OH}$ compared to $\mathrm{CO}$ in several nearby ULIRGs. According to González-Alfonso et al. (2017), this could suggest that $\mathrm{OH}$ and $\mathrm{CO}$ trace outflows in different regions of the galaxy. After all, they argue that as the outflowing gas expands and breaks up into clumps, it will "cover a decreasing fraction of the far-IR continuum". Unlike the CO, the $\mathrm{OH}$ molecules are radiatively excited, and hence "produce lower absorption and emission" the further out the gas flows (González-Alfonso et al. 2017). This scenario may also explain the fact that the molecular mass outflow rates that we derive from $\mathrm{H}_{2}$ appear on average lower than those based on $\mathrm{OH}$ studies.

Our results suggest that consensus has yet to be reached on how effective molecular gas outflows are in influencing the star formation properties of nearby ULIRGs. We argue that detailed $3 \mathrm{D}$-imaging of the different gas phases is a valuable tool to help determine the evolutionary role of multi-phase gas outflow in the evolution of these IR-luminous galaxies.

\section{Conclusions}

We presented the detection and characterization of massive outflows of hot molecular gas in three out of four nearby ULIRGs. We imaged the molecular outflows at sub-kpc resolution with VLT-SINFONI, using the $\mathrm{H}_{2} 1-0 \mathrm{~S}$ (1) $2.12 \mu \mathrm{m}$ line. We derive the following conclusions:

- The $\mathrm{H}_{2}$ outflows spread from the nuclear region out to scales of $0.7-1.6 \mathrm{kpc}$.

- The $\mathrm{H}_{2}$ outflows contain a hot molecular gas mass of $M_{\mathrm{H} 2 \text { (hot) }} \sim 6-8 \times 10^{3} M_{\odot}$. Assuming a hot-to-cold $\mathrm{H}_{2}$ ratio of $6 \times 10^{-5}$, as previously found in outflows from LIRGs, this corresponds to total molecular gas masses of $M_{\mathrm{H} 2} \sim 10^{8} M_{\odot}$.

- The corresponding total mass outflow rates of molecular gas are $\dot{M}_{\mathrm{H} 2} \sim 30-85 M_{\odot} \mathrm{yr}^{-1}$, which is a factor of five to ten lower than the star formation rates in these systems.

- The typical outflow velocities range from $300-500 \mathrm{~km} \mathrm{~s}^{-1}$, which suggests that a large fraction of outflowing gas remains gravitationally bound and is merely re-distributed within the ULIRGs. 
B. H. C. Emonts et al.: Outflows of hot molecular gas in ULIRGs

- The fastest outflow is associated with the Compton-thick AGN candidate IRAS 14348-1447, with a velocity-wing in the emission-line profile that stretches out to $\sim 900 \mathrm{~km} \mathrm{~s}^{-1}$ at zero intensity.

We do not find convincing evidence that the molecular outflows that we discovered in the $\mathrm{H}_{2} 2.12 \mu \mathrm{m}$ line in three of the nearest ULIRGs dramatically change the evolution of these systems. Future studies with JWST and ALMA will provide the sensitivity to further investigate how common and how destructive molecular outflows are in ULIRGs throughout the Universe.

Acknowledgements. We thank the anonymous referee for valuable feedback that helped improve the paper. The research leading to these results was funded by the Spanish Ministerio de Economía y Competitividad (MINECO) under grants AYA2012-32295, ESP2015-68964-P, AYA2016-76682-C3-2-P, and AYA201554346-C2-1-P. Based on observations collected at the European Organisation for Astronomical Research in the Southern Hemisphere, Chile, progs. 077.B-0151A and 081.B-0042A. Based on observations made with the NASA/ESA Hubble Space Telescope, and obtained from the Hubble Legacy Archive, which is a collaboration between the Space Telescope Science Institute (STScI/NASA), the Space Telescope European Coordinating Facility (ST-ECF/ESA), and the Canadian Astronomy Data Centre (CADC/NRC/CSA). The National Radio Astronomy Observatory is a facility of the National Science Foundation operated under cooperative agreement by Associated Universities, Inc.

\section{References}

Aalto, S., Garcia-Burillo, S., Muller, S., et al. 2015, A\&A, 574, A85 Alatalo, K. 2015, ApJ, 801, L17

Alonso-Herrero, A., Poulton, R., Roche, P. F., et al. 2016, MNRAS, 463, 2405

Arribas, S., \& Colina, L. 2003, ApJ, 591, 791

Arribas, S., Colina, L., Alonso-Herrero, A., et al. 2012, A\&A, 541, A20

Arribas, S., Colina, L., Bellocchi, E., Maiolino, R., \& Villar-Martin, M. 2014 A\&A, 568, A14

Bellocchi, E., Arribas, S., Colina, L., \& Miralles-Caballero, D. 2013, A\&A, 557, A59

Boomsma, R., Oosterloo, T. A., Fraternali, F., van der Hulst, J. M., \& Sancisi, R. 2005, A\&A, 431, 65

Calzetti, D., Armus, L., Bohlin, R. C., et al. 2000, ApJ, 533, 682

Caputi, K. I., Dole, H., Lagache, G., et al. 2006, A\&A, 454, 143

Cazzoli, S., Arribas, S., Colina, L., et al. 2014, A\&A, 569, A14

Cazzoli, S., Arribas, S., Maiolino, R., \& Colina, L. 2016, A\&A, 590, A125

Chung, A., Yun, M. S., Naraynan, G., Heyer, M., \& Erickson, N. R. 2011, ApJ, 732, L15

Cicone, C., Maiolino, R., Sturm, E., et al. 2014, A\&A, 562, A21

da Cunha, E., Charmandaris, V., Díaz-Santos, T., et al. 2010, A\&A, 523, A78

Dale, D. A., Sheth, K., Helou, G., Regan, M. W., \& Hüttemeister, S. 2005, AJ, 129,2197

Dasyra, K. M., \& Combes, F. 2011, A\&A, 533, L10

Dasyra, K. M., \& Combes, F. 2012, A\&A, 541, L7

Dasyra, K. M., Combes, F., Novak, G. S., et al. 2014, A\&A, 565, A46

Dasyra, K. M., Bostrom, A. C., Combes, F., \& Vlahakis, N. 2015, ApJ, 815, 34

Davies, R. I., Sternberg, A., Lehnert, M., \& Tacconi-Garman, L. E. 2003, ApJ, 597, 907

Elvis, M., Wilkes, B. J., McDowell, J. C., et al. 1994, ApJS, 95, 1

Emonts, B. H. C., Morganti, R., Tadhunter, C. N., et al. 2005, MNRAS, 362, 931

Emonts, B. H. C., Piqueras-López, J., Colina, L., et al. 2014, A\&A, 572, A40

Evans, A. S., Mazzarella, J. M., Surace, J. A., \& Sanders, D. B. 2002, ApJ, 580,

Feruglio, C., Maiolino, R., Piconcelli, E., et al. 2010, A\&A, 518, L155

Feruglio, C., Fiore, F., Carniani, S., et al. 2015, A\&A, 583, A99

Fischer, J., Sturm, E., González-Alfonso, E., et al. 2010, A\&A, 518, L41

Fitzpatrick, E. L. 1999, PASP, 111, 63

Franceschini, A., Braito, V., Persic, M., et al. 2003, MNRAS, 343, 118

Fraternali, F., \& Binney, J. J. 2006, MNRAS, 366, 449

García-Burillo, S., Combes, F., Usero, A., et al. 2015, A\&A, 580, A35

García-Marín, M., Colina, L., \& Arribas, S. 2009, A\&A, 505, 1017

González-Alfonso, E., Fischer, J., Spoon, H. W. W., et al. 2017, ApJ, 836, 11
Guillard, P., Ogle, P. M., Emonts, B. H. C., et al. 2012, ApJ, 747, 95

Guillard, P., Boulanger, F., Lehnert, M. D., Appleton, P. N., \& Pineau des Forêts, G. 2015, SF2A-2015: Proc. Annual Meeting of the French Society of Astronomy and Astrophysics, eds. F. Martins, S. Boissier, V. Buat, L. Cambrésy, \& P. Petit, 81

Heckman, T. M., Armus, L., \& Miley, G. K. 1990, ApJS, 74, 833

Hill, M. J., \& Zakamska, N. L. 2014, MNRAS, 439, 2701

Ho, L. C. 2008, ARA\&A, 46, 475

Indebetouw, R., Mathis, J. S., Babler, B. L., et al. 2005, ApJ, 619, 931

Iwasawa, K., Sanders, D. B., Teng, S. H., et al. 2011, A\&A, 529, A106

Le Floc'h, E., Papovich, C., Dole, H., et al. 2005, ApJ, 632, 169

Lehnert, M. D., \& Heckman, T. M. 1996, ApJ, 462, 651

Magnelli, B., Popesso, P., Berta, S., et al. 2013, A\&A, 553, A132

Mahony, E. K., Morganti, R., Emonts, B. H. C., Oosterloo, T. A., \& Tadhunter, C. 2013, MNRAS, 435 , L58

Markwardt, C. B. 2009, Astronomical Data Analysis Software and Systems XVIII, eds. D. A. Bohlender, D. Durand, \& P. Dowler, ASP Conf. Ser., 411, 251

Martin, C. L. 2006, ApJ, 647, 222

Mazzalay, X., Saglia, R. P., Erwin, P., et al. 2013, MNRAS, 428, 2389

Medling, A. M., Vivian, U., Guedes, J., et al. 2014, ApJ, 784, 70

Medling, A. M., Vivian, U., Rich, J. A., et al. 2015, MNRAS, 448, 2301

Morganti, R., Oosterloo, T. A., Tadhunter, C. N., van Moorsel, G., \& Emonts, B. 2005, A\&A, 439, 521

Morganti, R., Fogasy, J., Paragi, Z., Oosterloo, T., \& Orienti, M. 2013, Science, 341, 1082

Morganti, R., Veilleux, S., Oosterloo, T., Teng, S. H., \& Rupke, D. 2016, A\&A, 593, A30

Narayanan, D., Cox, T. J., Kelly, B., et al. 2008, ApJS, 176, 331

Nardini, E., Risaliti, G., Watabe, Y., Salvati, M., \& Sani, E. 2010, MNRAS, 405, 2505

Novak, M., Smolcic, V., Delhaize, J., et al. 2017, A\&A, 602, A5

Ogle, P., Boulanger, F., Guillard, P., et al. 2010, ApJ, 724, 1193

Ogle, P., Davies, J. E., Appleton, P. N., et al. 2012, ApJ, 751, 13

Oosterloo, T., Fraternali, F. \& Sancisi, R. 2007, AJ, 134, 1019

Pereira-Santaella, M., Colina, L., García-Burillo, S., et al. 2016, A\&A, 594, A81

Pérez-González, P. G., Rieke, G. H., Egami, E., et al. 2005, ApJ, 630, 82

Piqueras López, J., Colina, L., Arribas, S., Alonso-Herrero, A., \& Bedregal, A. G. 2012, A\&A, 546, A64

Piqueras López, J., Colina, L., Arribas, S., \& Alonso-Herrero, A. 2013, A\&A, 553, A85

Rieke, G. H., \& Lebofsky, M. J. 1985, ApJ, 288, 618

Rigopoulou, D., Kunze, D., Lutz, D., Genzel, R., \& Moorwood, A. F. M. 2002, A\&A, 389, 374

Rodríguez Zaurín, J., Tadhunter, C. N., Rose, M., \& Holt, J. 2013, MNRAS, 432, 138

Rupke, D. S. N., \& Veilleux, S. 2011, ApJ, 729, L27

Rupke, D. S. N., \& Veilleux, S. 2013a, ApJ, 768, 75

Rupke, D. S. N., \& Veilleux, S. 2013b, ApJ, 775, L15

Rupke, D. S. N., \& Veilleux, S. 2015, ApJ, 801, 126

Rupke, D. S., Veilleux, S., \& Sanders, D. B. 2005, ApJS, 160, 115

Sakamoto, K., Aalto, S., Combes, F., Evans, A., \& Peck, A. 2014, ApJ, 797, 90

Sanders, D. B., \& Mirabel, I. F. 1996, ARA\&A, 34, 749

Scoville, N. Z., Hall, D. N. B., Ridgway, S. T., \& Kleinmann, S. G. 1982, ApJ, 253, 136

Scoville, N. Z., Evans, A. S., Thompson, R., et al. 2000, AJ, 119, 991

Soifer, B. T., \& Neugebauer, G. 1991, AJ, 101, 354

Soto, K. T., \& Martin, C. L. 2012, ApJS, 203, 3

Spoon, H. W. W., Farrah, D., Lebouteiller, V., et al. 2013, ApJ, 775, 127

Sturm, E., González-Alfonso, E., Veilleux, S., et al. 2011, ApJ, 733, L16

Tadhunter, C., Morganti, R., Rose, M., Oonk, J. B. R., \& Oosterloo, T. 2014, Nature, 511, 440

Valentijn, E. A., \& van der Werf, P. P. 1999, ApJ, 522, L29

van der Werf, P. P., Genzel, R., Krabbe, A., et al. 1993, ApJ, 405, 522

Veilleux, S., Kim, D.-C., \& Sanders, D. B. 1999, ApJ, 522, 113

Veilleux, S., Melendez, M., Sturm, E., et al. 2013, ApJ, 776, 27

Veilleux, S., Meléndez, M., Tripp, T. M., Hamann, F., \& Rupke, D. S. N. 2016, ApJ, 825, 42

Wagner, A. Y., Bicknell, G. V., \& Umemura, M. 2012, ApJ, 757, 136

Westmoquette, M. S., Clements, D. L., Bendo, G. J., \& Khan, S. A. 2012 MNRAS, 424, 416

Zubovas, K., \& King, A. R. 2014, MNRAS, 439, 400 


\section{Appendix A: Individual sources}

In the following sections we provide detailed information regarding the broad component $\mathrm{H}_{2}$ emission in IRAS 12112+0305, IRAS 14348-1447, IRAS 17208-0014, and IRAS 22491-1808.

\section{A.1. IRAS $12112+0305$}

The broad-component emission is seen in the NE galaxy of this galaxy-pair. The broad-component emission is blueshifted and found in the region where the narrow-component disk is redshifted or at the systemic velocity. The broad-component emission appears to originate from the nuclear region, is only seen on one side of the nucleus, and stretches out to $\sim 1.6 \mathrm{kpc}$. The narrow-component disk shows a $0 \mathrm{~km} \mathrm{~s}^{-1}$ iso-velocity contour, which position angle (PA) changes from $\mathrm{PA} \sim 0^{\circ}$ to $\mathrm{PA} \sim 23^{\circ}$. We argue that the latter is the most likely PA of the minor axis of the galaxy, given that it is perpendicular to the elongated morphology of the galaxy in the HST/NICMOS imaging (Fig. 6). This means that the outflow spans a cone that is oriented about $15-55^{\circ}$ off the minor axis. The outflow peaks in intensity and velocity roughly $\sim 0.5 \mathrm{kpc}$ from the core. Here the outflow has a bulk velocity of $\sim 200 \mathrm{~km} \mathrm{~s}^{-1}$ with respect to the systemic velocity, which it maintains out to a distance of $\sim 1.6 \mathrm{kpc}$. The wing of the broad component stretches out to $-700 \mathrm{~km} \mathrm{~s}^{-1}$ at zerointensity. Table 1 gives more details. Figure 2 suggests that the SW galaxy of this ULIRG also shows faint broad-component emission, but Fig. A.1 clarifies that this merely reflects our uncertainty in the Gaussian fitting procedure due to the underlying strong continuum emission of this source.

\section{A.2. IRAS $14348-1447$}

The broad-component emission is seen in the SW galaxy of this galaxy-pair, which hosts an AGN that is likely to be Comptonthick (Iwasawa et al. 2011). The broad component shows the most blueshifted emission of the four galaxies in our sample, stretching out to $-900 \mathrm{~km} \mathrm{~s}^{-1}$ at zero-intensity. Previous work showed that gas outflows are on average faster in the presence of an AGN (Spoon et al. 2013; Arribas et al. 2014; Cicone et al. 2014; Hill \& Zakamska 2014; see also models by Narayanan et al. 2008). The fact that this is also the case when comparing IRAS 14348-1447 with the other ULIRGs in our sample strongly suggests that the outflow in IRAS 14348-1447 originates from the nuclear region. The broad component is resolved only marginally in a westerly direction, with a size of $\sim 0.9 \mathrm{kpc}$. However, the fact that the narrow-component emission is close to the systemic velocity throughout the disk suggests that the galaxy disk is aligned close to face-on. This makes our estimated PA $\sim-55^{\circ}$ for the minor axis, as based on the $0 \mathrm{~km} \mathrm{~s}^{-1}$ iso-velocity contour and HST/NICMOS morphology, rather uncertain. Nevertheless, if the outflow traced by the broad component were aligned along the minor axis of the galaxy, it would mostly propagate along our line of sight, which means that the true extent and velocity of the broad-component emission may be significantly larger. More details are given in Table 1.

\section{A.3. IRAS 17208-0014}

IRAS 17208-0014 has been associated with multi-phase gas outflows by various authors (Rupke et al. 2005; Martin 2006; Sturm et al. 2011; Arribas et al. 2014; Soto \& Martin 2012; Medling et al. 2015). However, several integral-field studies did not

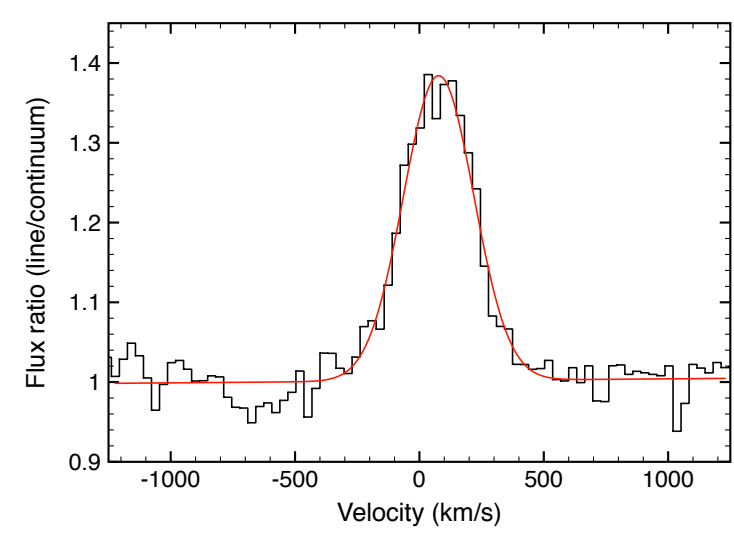

Fig. A.1. Spectrum of the $\mathrm{H}_{2} 1-0 \mathrm{~S}(1)$ line in the SW galaxy of the IRAS 12112+0305 galaxy-pair, that is, the galaxy that does not host the prominent outflow. Plotted on the $y$-axis is the ratio of the emission-line flux and the continuum flux. The spectrum is extracted from a region of five pixels (three pixels both $\mathrm{E}$ to $\mathrm{W}$ and $\mathrm{N}$ to $\mathrm{S}$ ), centered on the peak of the continuum emission. The red line shows a single Gaussian function, which accurately fits the $\mathrm{H}_{2}$ line. This plot shows that the weak broadcomponent emission from Fig. 2 is merely a result of our uncertainty in the Gaussian fitting procedure due to the underlying strong continuum emission of this source.

find evidence for such outflows in the ionized phase (Arribas \& Colina 2003; Westmoquette et al. 2012; Rupke \& Veilleux 2013a), and Arribas \& Colina (2003) even argued the presence of gas inflows. In our SINFONI data, the overall $\mathrm{H}_{2}$ kinematics appear to follow the $\mathrm{CO}$ kinematics of cold molecular gas discussed in detail by García-Burillo et al. (2015). The broadcomponent $\mathrm{H}_{2}$ emission is only seen near the systemic velocity, and spreads across $\sim 2 \mathrm{kpc}$, which is a substantial fraction of the narrow-component disk. In Sect. 3.2, we discuss that this broadcomponent emission is not likely to be related to an outflow, although its true nature remains unclear.

We do not detect a hot $\mathrm{H}_{2}$ counterpart to the cold molecular outflow previously discovered in CO by García-Burillo et al. (2015). As shown in Sect. 3.3, we derive a firm upper limit on the outflow of hot molecular $\mathrm{H}_{2}$ gas of $M_{\mathrm{H} 2 \text {-hot }} \lesssim 400 M_{\odot}$, which results in a hot-to-cold molecular gas mass ratio of $\lesssim 9 \times 10^{-6}$.

\section{A.4. IRAS 22491-1808}

The broad-component emission is seen in the E galaxy of this galaxy pair. The broad component is most prominent and blueshifted west of the nucleus. It spans a cone that is aligned roughly $10-65^{\circ}$ off the minor axis of the narrow-component disk, which we estimate has $\mathrm{PA} \sim 50^{\circ}$, based on the $0 \mathrm{~km} \mathrm{~s}^{-1}$ isovelocity contour. A faint redshifted counterpart could be present towards the south, but this needs to be verified. The broadcomponent emission stretches $0.7 \mathrm{kpc}$ from the nucleus, where it also shows the highest bulk velocities of roughly $-100 \mathrm{~km} \mathrm{~s}^{-1}$ with respect to the systemic velocity. The maximum velocity of the outflow reaches only $-500 \mathrm{~km} \mathrm{~s}^{-1}$ at zero-intensity. Table 1 and Sect. 3 give more details.

\section{Appendix B: Velocity dispersion maps}

Figure B.1 shows the velocity dispersion of the broadcomponent emission from Figs. 2-5. 
B. H. C. Emonts et al.: Outflows of hot molecular gas in ULIRGs
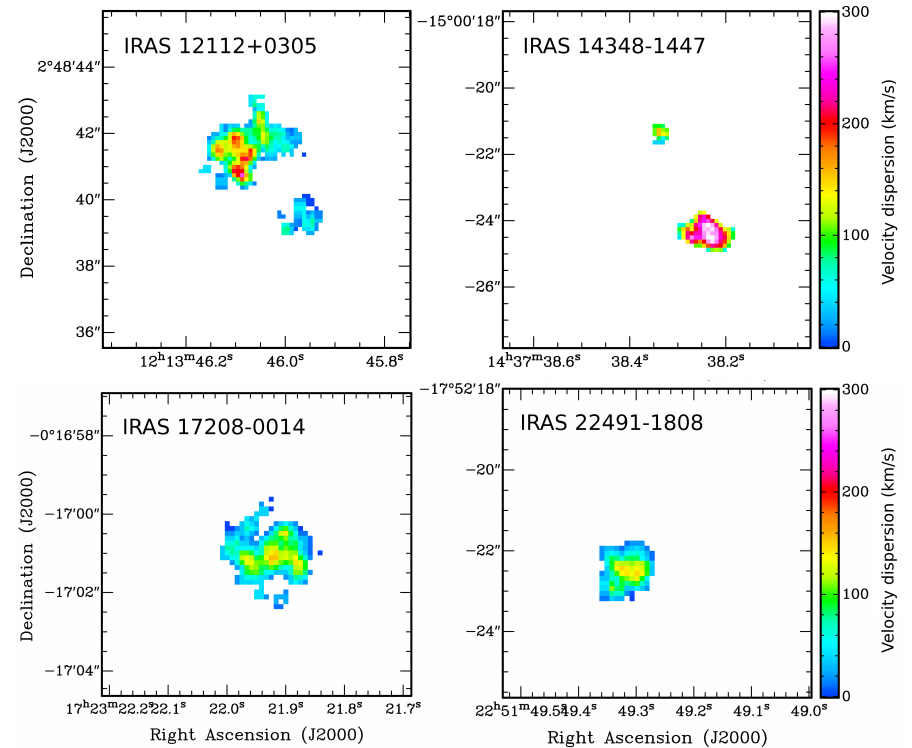

Fig. B.1. Maps of the velocity dispersion, $\sigma=F W H M / 2.35$, of the broad-component $\mathrm{H}_{2} 1-0 \mathrm{~S}(1)$ emission in IRAS 12112+0305, IRAS 14348-1447, IRAS 17208-0014, and IRAS 22491-1808. The velocity scaling is the same in all four plots. 